\title{
Hacia una cultura de seguridad del paciente: el futuro de la profesión de enfermería
}

Towards a Culture of Patient Safety:

The Future of the Nursing Profession

Rumo a uma cultura de segurança do paciente:

o futuro da profissão de enfermagem

Manuel Lillo-Crespo'

DOI: 10.5294/aqui.2017.17.4.1

La creación de una cultura centrada en la seguridad del paciente en los diferentes contextos de salud y enfermedad supone un leitmotif para el desarrollo de enfermería como profesión a nivel global en las sociedades actuales. Las estrategias sanitarias impulsadas hoy día por entidades como la Organización Mundial de la Salud (OMS) o la Comisión Europea en materia de seguridad del paciente tienen su base en objetivos globales como el empoderamiento del usuario de los sistemas sanitarios para la toma de decisiones sobre su propia salud y enfermedad, el desarrollo de buenas prácticas basadas en evidencias, así como la innovación a través de dispositivos que garanticen la seguridad de unos cuidados centrados en la persona e individualizados. En todos ellos está presente el enfoque de seguridad para el usuario. No hay más que observar las convocatorias de investigación, desarrollo e innovación publicadas por estas organizaciones, también por los gobiernos de las naciones, e incluso por compañías privadas del sector salud para corroborar que el concepto seguridad ha cobrado un matiz transversal a cualquier otro tema, y se ha convertido en un requisito indispensable que debe estar presente, directa 0 indirectamente, en todas las iniciativas.

Sin embargo, el concepto "seguridad del paciente" no deja de ser el planteamiento positivo de otra noción que genera desagrado entre los profesionales de la salud, como es el "error", y cuya connotación es obviamente negativa. Las culturas de seguridad

1 Universidad de Alicante, España. Manuel.Lillo@ua.es del paciente no solo deben estar orientadas a la corrección del error, sino a la prevención del mismo, dadas sus consecuencias para la salud de las personas. Curiosamente, si estableciéramos un paralelismo entre ambos conceptos y los paradigmas de atención sanitaria actuales podríamos decir que el clásico paradigma biomédico, centrado en la patología y su curación es directamente proporcional al concepto de "error" y a la acción de "curar", mientras que los conceptos de "seguridad del paciente" y "prevención de eventos adversos" forman parte de un nuevo paradigma centrado en la salud y no en la enfermedad, más propio de la profesión enfermera. Es en dicha transformación del paradigma de atención a las personas en la que la profesión de enfermería emerge como agente de cambio indispensable. Por otra parte, el desarrollo de estas iniciativas que sitúan al usuario en el centro de las prioridades de los sistemas sanitarios mundiales, y a la salud como su máximo exponente, paralelamente ha posicionado a la figura de enfermería en un puesto clave para la toma de decisiones dentro del equipo de salud. Pero, ¿es consciente realmente el profesional de enfermería de cuál es el paradigma que favorece su propio desarrollo y de las oportunidades que tiene ante sí?

Prácticamente toda la bibliografía que se ha publicado al respecto procede del mundo anglosajón-estadounidense y aboga por un concepto multidisciplinar, interprofesional y centrado en el paciente. El Institute for Healthcare Improvement (IHI) y la Joint Commission, en concreto a través de su Portal de Transiciones en el Cuidado (ToC Portal - Transitions of Care Portal) han sido sus 
máximos exponentes, y han lanzado vasta producción documental que ha servido de guía en diferentes contextos a nivel mundial. También se han implementado en la práctica clínica nuevos roles profesionales como el Health Coach y el Patient Experience Officer, que desde el Reino Unido y Estados Unidos, respectivamente, han aunado competencias profesionales relacionadas con la seguridad del paciente y todo lo que ello conlleva en materia de calidad y satisfacción. Sin embargo, no son muchos los contextos iberoamericanos donde las culturas de seguridad del paciente se han desarrollado y han sido, en el mejor de los casos, iniciativas con un enfoque marcadamente clínico y relacionadas con especialidades médicas como la Medicina Preventiva, en un intento por aterrizar el concepto de seguridad del paciente en un paradigma de atención biomédica clásico basado en la patología y alejado de la creación de auténticas culturas transformadoras. No obstante, ¿entiende el mundo iberoamericano el concepto de seguridad del paciente de la misma forma que lo hace el anglosajón? ¿Cuál es el papel de enfermería? ¿Hasta qué punto son útiles los documentos creados en contextos distintos?

Posicionamientos específicos de enfermería en esta línea que requieran mención son: las contribuciones de la enfermera doctora Linda Aiken del Centre for Health Outcomes and Policy Research, de la Escuela de Enfermería en la Universidad de Penn, en Filadelfia, quien en su numerosa bibliografía publicada, y de gran calado social, ha aportado claras evidencias científicas que correlacionan índices de mortalidad hospitalaria, datos sobre infecciones nosocomiales, ratios enfermera-paciente, clima o entorno laboral, seguridad del paciente y tasas de satisfacción laboral del personal de enfermería, donde demuestra que todos ellos redundan en la calidad de la institución, y se convierten en aspectos clave de la satisfacción del usuario $(1,2)$. Por no olvidar sus contribuciones sobre los denominados magnet hospitals, centros sanitarios que han demostrado tener mejores ambientes de trabajo y más seguros, personal de enfermería más preparado, con una ratio de enfermera-paciente aceptable de acuerdo con las cargas de trabajo, y mayor colaboración enfermero-médico, por lo que han obtenido mejores resultados en salud (3). Por otra parte, la también enfermera doctora Afaf Meleis, reconocida investigado- ra y teórica, ha contribuido con el desarrollo de su teoría de las transiciones para dar explicación a través de la investigación a estos temas (4).

En los últimos años en Europa, y a partir de las recomendaciones de la OMS en cuanto a seguridad del paciente y prevención de errores en la práctica profesional, ya se está trabajando en iniciativas como la comenzada en 2014 denominada Healthcare Improvement Science (HIS), a través del Proyecto Europeo Improvement Science Training for European Healthcare Workers (ISTEW) financiado por la Comisión Europea y en el que participó España $(5,6)$. En la actualidad, la inquietud de los países europeos por continuar mejorando la calidad asistencial de sus instituciones - mayoritariamente públicas a diferencia de Estados Unidos, y sin perder de vista la seguridad del usuario — se ha materializado en esta iniciativa que incluye también una formación específica de los profesionales de la salud y que define a la ciencia de las mejoras en cuidados de salud (healthcare improvement science) como: "la generación de conocimiento para cultivar el cambio y ofrecer una atención centrada en la persona que sea segura, eficaz, eficiente, equitativa y oportuna. Implica la mejora en los resultados del paciente, el desempeño de los sistemas sanitarios y la salud de la población" (7). Como novedad, esta definición nos hace traspasar las puertas de las instituciones sanitarias y nos conecta con las comunidades y las propias familias, siendo estas uno de los pilares de las sociedades iberoamericanas. En esta línea, la contribución del aprendizaje experiencial y la evidencia procedente de la práctica de los cuidadores, especialmente familiares, tan presentes en las sociedades iberoamericanas, ha dado pie al desarrollo de manuales de buenas prácticas donde la seguridad del paciente va más allá de la omisión de daño, y se articula como una iniciativa que garantice los mejores cuidados posibles y con la mayor calidad para aquellos a quienes van destinados. Trabajar en seguridad del paciente supone reconocer aquellas prácticas basadas en la experiencia cuando no existen evidencias científicas. Proyectos como Palliare, también bajo el paraguas de la Comisión Europea, ponen de manifiesto la seguridad y las buenas prácticas en el todavía desconocido campo de los cuidados ante la demencia $(8,9)$. 


\section{Referencias}

1. Aiken LH, Sermeus W, van den Heede K, Sloane DM, Busse R, McKee M. Patient safety, satisfaction, and quality of hospital care: Cross sectional surveys of nurses and patients in 12 countries in Europe and the United States. BMJ. 2012;344:e1717.

2. Aiken LH, Sloane DM, van den Heede K, Busse R, Diomidous M, Kinnunen J. Nurse staffing and education and hospital mortality in nine European countries: A retrospective observational study. Lancet. 2014;383(9931):1824-30.

3. Kelly LA, McHugh MD, Aiken LH. Nurse outcomes in magnet ${ }^{\circledR}$ and non-magnet hospitals. J Nurs Adm. 2011;41(10):428-33.

4. Meleis AI. Transitions theory: Middle range and situation specific theories in nursing research and practice. New York: Springer Publishing Company; 2010.

5. MacRae R, Rooney KD, Taylor A, Ritters K, Sansoni J, Lillo M, et al. Making it easy to do the right thing in healthcare: Advancing improvement science education through accredited pan European higher education modules. Nurs Educ Today. 2016;42:41-6.

6. Istew Project [sede Web]. University of the West of Scotland; [Visitado 2017 jun 14]. Disponible en: http://www.uws. ac.uk/improvementscience/

7. Skela-Savič B, MacRae R, Lillo-Crespo M, Rooney KD. The development of a consensus definition for healthcare improvement science (HIS) in seven European countries: A consensus methods approach. Sloven J Public Health. 2016;56(2):82-90.

8. Tolson D, Holmerova I, Macrae R, Waugh A, Hvalič-Touzery S, de Abreu W, et al. Improving advanced dementia care: An interprofessional palliare learning framework. JAMDA. 2017;18(7):561-3.

9. Palliare Project [sede Web]. University of the West of Scotland; [Visitado 2017 jun 14]. Disponible en: https://www.uws. ac.uk/palliareproject/ 